\title{
Accountability for Reasonableness, for Priority Setting and Resource Allocation: A Case Scenario
}

\author{
Zoheb Rafique \\ Lecturer Department of Biochemistry \\ Liaquat University of Medical \& Health Sciences (LUMHS), \\ Jamshoro, Pakistan \\ Email: dr_zohaib@hotmail.com
}

\begin{abstract}
The purpose of this paper is to discuss the accountability for reasonableness and its four conditions. This explains the priority setting and resource allocation for scarce resources. In this article it is discussed that how the scarce resources in a developing country like Pakistan be allocated in health care. This is explained with the help of case scenario.
\end{abstract}

Key Words: Accountability, Reasonableness, Priority Setting, Resource Allocation

INTRODUCTION: Pakistan as a developing country has very limited health care resources when considering a huge population of over 180 million. We have very few tertiary care hospitals and they are providing services to the whole country. Majority of people in our country are poor and they are unable to afford the expenses of private hospitals, though private hospitals are also very few. Thousands of doctors are unemployed and still we have shortage of doctors in Public Sector. Majority of BHU (Basic Health Units) are closed as majority of doctors belong to urban areas and they don't want to work in remote village areas. In all these situations, it is very difficult to maintain health care throughout country. In this paper, I will take into account four conditions of accountability for reasonableness for priority setting and resource allocation. I will consider a tertiary care hospital scenario where I did my house job in medicine ward a few years ago. There were majority of patients suffering from chronic liver diseases (CLD).

DISCUSSION: Before discussing the four conditions of accountability for reasonableness, I will briefly discuss the case scenario. In my medicine ward as I earlier said majority of patients were of chronic liver diseases (CLD) and it includes Hepatitis B, Hepatitis C and cirrhosis of liver. Cirrhosis is the end result of hepatocellular injury that leads to both fibrosis and nodular regeneration throughout the liver. The clinical features result from hepatic cell dysfunction, Porto-systemic shunting, and Portal hypertension. Cirrhosis may cause no symptoms for long periods. One of the major complications is upper gastrointestinal tract bleeding which may occur from varices, portal hypertensive gastropathy, or gastroduodenal ulcer. Hemorrhage may be massive, resulting in fatal exsanguinations. Esophageal varices are found in $50 \%$ of patients with cirrhosis. There are several treatment and management options available for esophageal varices including acute resuscitation as initial management, pharmacologic therapy, balloon tube tamponade, portal decompressive procedures and emergent endoscopy. Endoscopic techniques are also used for prevention of rebleeding ${ }^{1}$. Now, I will discuss my case scenario. In our hospital there was one professor of Medicine who was trained in doing endoscopy and Wednesday was fixed for performing endoscopies. Emergent endoscopy is performed after the patient's hemodynamic status has been appropriately stabilized (usually within 2-12 hours). Majority of poor patients come to hospital in end stage liver diseases. I have taken this case scenario as it is a perfect example of scarce resources. Many patients faced problems as their endoscopies were not performed on time as there was only one day fixed in a week and the reason was the Professor was busy and though he was training others in the meantime (Every Wednesday). Now I will apply the four conditions of accountability for reasonableness for priority setting and resource allocation. These four conditions are publicity condition, relevance condition, revisions and appeals condition and regulative condition. Accountability for reasonableness makes it possible to educate all stakeholders about the substance of deliberation about fair decisions under resource constraints. It facilitates social learning about limits. It connects decision making in healthcare institutions to broader, more fundamental democratic deliberative processes ${ }^{2}$. In my case scenario I will apply the four conditions as follows. The first one is publicity condition. It states that decisions regarding limits to care and their rationales must be publicly accessible to clinicians, patients, and citizens in a publicly administered system. When the patients suffer the complication of esophageal varices, they are informed about the limited capacity of the ward to arrange endoscopy as it is done on only Wednesdays and surgical ward have their own burden of patients to be done endoscopies, due to this reason we were unable to send patients to surgical wards and the patients and their relatives mostly agrees on this setup and if 
any emergency happens, only then we take help from surgical ward or send the patients to any other hospital, so the first condition is fulfilled. In above scenario second condition is also fulfilled which is relevance condition. It states that the reasons for limit-setting decisions will be reasonable if it appeals to evidence, reason, and principles that are accepted as relevant by fair-minded people who are disposed to finding mutually justifiable terms of cooperation. In my case scenario the decision making is according to the framework. The rationales were reasonable as it is evident that we had limited facility of endoscopy and it was fairly accepted by patients and their relatives and also by doctors and other hospital staff. In our setup priority was given to those patients who needed emergency endoscopy rather than those who requires endoscopy for diagnostic procedures. The third condition is revisions and appeals condition. This condition is a very common problem in government hospitals and in our scenario we request consultants from surgical ward to do emergency endoscopy if we think patient is serious and he or she may die if the endoscopy is not performed on time or in other case the other hospital is very far so that it will be late if we send the patient to other setup and here comes the function of on call consultants also, the on call consultants plays huge role in these emergency situations. This third condition is a mechanism for challenge and dispute resolution regarding limit setting decisions, including the opportunity for revising decisions in light of further evidence or arguments ${ }^{3}$. Thus we fulfill the third condition also by revising our decisions as I explained above. The fourth and last condition is regulative condition or enforcement. There is either voluntary or public regulation of the process to ensure that conditions 1-3 are met. This condition is also fulfilled in our setup as we communicate with the patient and their relatives about our limited resources. We are able to convince patients in our case scenario. The hospital leadership is constantly making efforts to meet the conditions of 'accountability for reasonableness' 4 .

CONCLUSION: In this paper I have discussed all four conditions of accountability for reasonableness, for priority setting and resource allocation. 'Accountability for reasonableness' is a framework that can be used to guide legitimate and fair priority setting in health care organizations, such as hospitals. In our beloved country Pakistan we have few government civil hospitals bearing the burden of millions of population. We try our best to serve the humanity. I am not claiming this system a perfect one, it needs a lot of improvement and the example is my case scenario in which we have very limited resources. Government should establish civil hospitals in small cities also and should increase their budget; they should recruit more doctors and nurses as we have shortage. They should train doctors with latest equipment and provide hospitals appropriate medicines. In addition to this all the hospitals should be provided with computers and also be made online so that a data system can be established and it can help the patients and also hospitals for future reference. The case scenario I took was from 2008 when I did my house job, but now I have been informed that the condition is further improving at our hospital because other Physicians are also trained in doing Endoscopies and they are sharing the burden of Cases. It must also be noted that our teaching hospital was upgraded as our college transformed into University in the previous decade and now we have more budget and more slots for the senior physicians who are working hard to make things better. We also have now increased capacity of postgraduate students. In mean while one private center is also working towards hepatic patients in our city and they are doing fine job and so sharing the burden and they have qualified gastroenterologists. I will conclude by saying that in such scarce resources our hospital (public sector) is doing fine job as it has to share the burden of millions of people because we have very few public sector hospitals in our country with the population of over 180 million.

\section{CONFICT OF INTEREST: No conflict of interest.}

\section{REFERENCES:}

1. Current Medical Diagnosis and Treatment. 2004; 43rd edition.

2. Norman Daniels. Accountability for reasonableness. BMJ. 2000; 321:1300- 1301.

3. D K Martin Et Al. Access to intensive care unit beds for neurosurgery patients: a qualitative case study. J. Neurol. Neurosurg. Psychiatry. 2003; 74: 1299-1303. 
Bangladesh Journal of Bioethics 2015; 6(2):23-25

4. Jennifer AH Bell Et Al. SARS and hospital priority setting: a qualitative case study and evaluation. BMC Health Services Research. 2004; 4:36. 